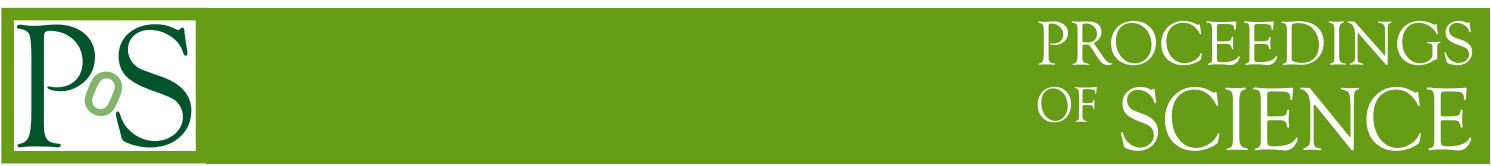

\title{
Distance to VY Canis Majoris with VERA
}

\section{Yoon Kyung Choi*}

Max-Planck-Institut fuer Radioastronomie

E-mail: ykchoi@mpifr-bonn.mpg.de

\section{Tomoya Hirota}

Mizusawa VERA Observatory, NAOJ

E-mail: tomoya.hirota@nao.ac.jp

\section{Mareki Honma}

Mizusawa VERA Observatory, NAOJ

E-mail: mareki.honma@nao.ac.jp

\section{Hideyuki Kobayashi}

Mizusawa VERA Observatory, NAOJ

E-mail: hideyuki.kobayashi@nao.ac.jp

We report on observational results of $\mathrm{H}_{2} \mathrm{O}$ and $\mathrm{SiO}(J=1-0, v=1$ and $v=2)$ masers around VY Canis Majoris (VY CMa) carried out with VERA for 13 months. Our astrometric monitoring measured a parallax of $0.88 \pm 0.08$ mas, and it corresponds to a distance of $1.14{ }_{-0.09}^{+0.11} \mathrm{kpc}$. This is the first trigonometric parallax measurement for VY CMa. Using our newly obtained distance with a high accuracy, the luminosity of VY CMa was re-estimated to be $(3 \pm 0.5) \times$ $10^{5} \mathrm{~L} \odot$. This improved luminosity is more consistent with the theoretical evolutionary model than previous values. Moreover, we considered 3-dimensional structure and kinematics of the circumstellar envelopes around VY CMa with proper motions and absolute positions of the $\mathrm{H}_{2} \mathrm{O}$ and $\mathrm{SiO}$ masers. The 3-dimensional structures and kinematics suggest a bipolar outflow around VY CMa along the line of sight.

The 9th European VLBI Network Symposium on The role of VLBI in the Golden Age for Radio Astronomy and EVN Users Meeting September 23-26, 2008

Bologna, Italy

\footnotetext{
* Speaker.
} 


\section{Introduction}

Red supergiants (RSGs), massive evolved stars, are important in the evolution of the Universe, for example, they provide heavy elements into the interstellar medium (ISM) through their strong stellar winds. Since RSGs are extremely rare due to the steepness of the initial mass function (IMF) and their short lifetime, our understanding is still poor in massive stellar evolution. Even their fundamental properties - luminosities, temperatures, ages, and initial masses - have large uncertainty because of possible errors in the estimated distances. Since most of RSGs are very far, it is difficult to obtain trigonometric parallaxes, which is the most reliable method for distance measurements.

VY Canis Majoris (VY CMa) is one of the most luminous red supergiants in our galaxy. There are also several estimations for luminosity and temperature of VY CMa (e.g., [14], [18], [15]), and they are based on a distance of $1.5 \mathrm{kpc}$ [13]. This previously accepted distance was obtained with assumption that the distance of VY CMa is the same with that of NGC 2362. However, the distance of the NGC 2362 star cluster was estimated with an accuracy of $30 \%$ [11] from a color-magnitude diagram. Since fundamental parameters of the star highly depend on its distance, an accurate distance determination is essential.

In addition, VY CMa has been known as a good example to study mass loss from massive evolved stars. VY CMa has strong maser emission in its circumstellar envelopes. Since $\mathrm{SiO}$ masers are known to be located at a few stellar radii and $\mathrm{H}_{2} \mathrm{O}$ masers are found further from the star, observations of those masers would reveal the circumstellar structure around massive evolved star. Furthermore, multi-epoch phase-referencing VLBI observations of masers enable us to study kinematics of the circumstellar envelopes.

Here, we describe distance measurements of VY CMa carried out with VERA, based on recently published paper [3], and preliminary results on the 3-dimensional structure and kinematics of the circumstellar envelopes around VY CMa.

\section{Observations and Data Analysis}

The VERA observations of $\mathrm{H}_{2} \mathrm{O}$ masers $\left(\mathrm{H}_{2} \mathrm{O} 6_{16}-5_{23}\right.$ transition, $\left.22.235080 \mathrm{GHz}\right)$ and $\mathrm{SiO}$ masers ( $\mathrm{SiO} J=1-0, v=1$ and 2 transitions, 43.122027 and $42.820584 \mathrm{GHz}$, respectively) around the red supergiant VY CMa were carried out for 13 months since 2006 April. We observed VY $\mathrm{CMa}$ and a position reference source J0725-2640 [12], separated by $1 .^{\circ} 059$, simultaneously with dual-beam [8]. The data were recorded onto magnetic tapes at a rate of $1024 \mathrm{Mbps}$, providing a bandwidth of $256 \mathrm{MHz}$, subdivided into $16 \mathrm{IF}$ channels of $16 \mathrm{MHz}$, with 2-bit digitization. During the $22 \mathrm{GHz}$ observations, one $16 \mathrm{MHz}$ IF channel was allocated to the $\mathrm{H}_{2} \mathrm{O}$ maser emission and the remaining $15 \mathrm{IF}$ channels were allocated to the continuum source. For the $43 \mathrm{GHz}$ observations, two $16 \mathrm{MHz}$ IF channels were assigned to the two $\mathrm{SiO}$ transitions and the other $14 \mathrm{IF}$ channels were assigned to the continuum source. The correlation was done at the Mitaka FX correlator [2], yielding a velocity resolution of $\sim 0.2 \mathrm{~km} \mathrm{~s}^{-1}$ for maser emission. Details on observations and correlation processing were described in [3].

All data reduction was conducted using AIPS. Amplitude and bandpass calibrations were made for the maser source (VY CMa) and continuum source (J0725-2640) independently. The flux den- 
sity of the reference source J0725-2640 was measured to be $185 \mathrm{mJy}$ at $22 \mathrm{GHz}$ with VERA, and was expected to be $150 \mathrm{mJy}$ at $43 \mathrm{GHz}$, which is not enough to detect fringes with VERA. Therefore, we applied fringe fitting solutions of the reference source (J0725-2640) to the target source (VY CMa) for phase-referencing at $22 \mathrm{GHz}$ in order to get absolute positions. However, the opposite way of application using phase solutions of the maser source (VY CMa) was preformed at $43 \mathrm{GHz}$. We assumed that an apparent motion of the reference source reflect that of the maser. We also applied the dual-beam phase calibration [8] to correct instrumental delay difference between the two beams, and we finally calibrated the tropospheric zenith delay offset [9]. After these calibrations, we obtained synthesized images to measure the positions with two-dimensional Gaussian fittings.

\section{Distance Measurements}

We measured positions of the $\mathrm{H}_{2} \mathrm{O}$ maser spot at the LSR velocity of $0.55 \mathrm{~km} \mathrm{~s}^{-1}$ in VY CMa using J0725-2640 as a position reference source over 13 months. Figure 1 shows the movements of the maser spot as a function of time (day of year) in right ascension and declination, respectively. Since the declination data have too large errors to estimate an annual parallax and a proper motion, we obtained a trigonometric parallax of VY CMa to be $0.88 \pm 0.08$ mas, corresponding to a distance of $1.14{ }_{-0.09}^{+0.11} \mathrm{kpc}$ using only the data in right ascension. Compared with the previously accepted distance [13], the distance of VY CMa became $76 \%$ of the previous estimate. As discussed in [4], [7], and [5], it is difficult to estimate the individual error sources quantitatively in VLBI astrometry. Therefore, we estimated errors of 0.17 mas in right ascension and 0.68 mas in declination from the standard deviations of the least-square analysis. The details on possible causes of the astrometric errors are discussed in [3].

With the newly determined distance, we re-estimated the luminosity of VY CMa to be $(3 \pm$ $0.5) \times 10^{5} \mathrm{~L}_{\odot}$. In order to compare with theoretical evolutionary tracks on the HR diagram, we used
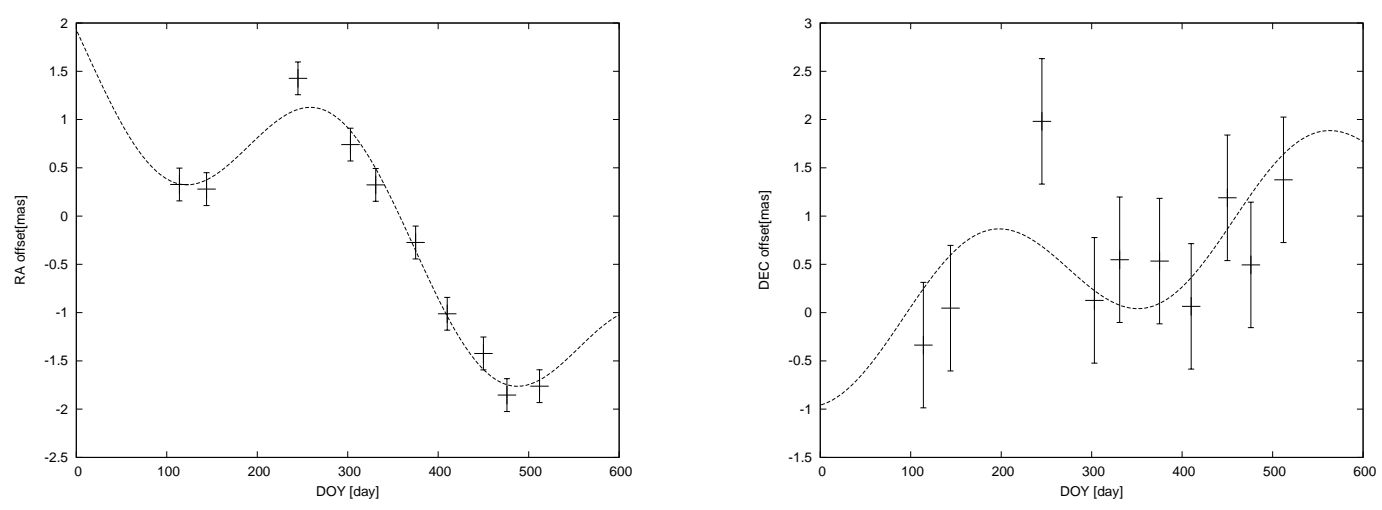

Figure 1: The positions of the $\mathrm{H}_{2} \mathrm{O}$ maser spot at the LSR velocity of $0.55 \mathrm{~km} \mathrm{~s}^{-1}$ in right ascension (left) and declination (right) against time (day of year since 2006 January 1). Points with error bars represent observed positions of the maser spot, and curves represent the best annual parallax and linear proper motion fit model. The position offsets are with respect to $\alpha(\mathrm{J} 2000.0)=07^{\mathrm{h}} 22^{\mathrm{m}} 58^{\mathrm{s}} .32906, \delta(\mathrm{J} 2000.0)=$ $-25^{\circ} 46^{\prime} 03^{\prime \prime} .1410$. 
temperature values from the literature. When we adopt a temperature of $3650 \mathrm{~K}$ [15], our result is consistent with the theoretical evolutionary track of an initial mass of $25 \mathrm{M}_{\odot}$ [16]. When we adopt $3000 \mathrm{~K}$ [18], the position on the HR diagram is still not consistent with the theoretical track (see figure 4 in [3]). However, it is much closer to the theoretically allowable zone than previous studies ([14], [18]). Since temperature does not depend on the distance, we cannot make a final conclusion with our results.

\section{Structure and Kinematics of the Circumstellar Envelopes}

As results of phase-referencing analyses, we obtained absolute positions of the $\mathrm{H}_{2} \mathrm{O}$ and $\mathrm{SiO}$ masers around VY CMa. Figure 2 (left) shows their distributions of three different masers. While the $v=1 J=1-0 \mathrm{SiO}$ masers constrain the stellar position with a ring-like structure, the $v=2 J=$ 1-0 SiO masers only trace a part of the circumstellar envelope because of their weak flux densities. Since $\mathrm{SiO}$ masers have been known to be located in the closest region from the stellar surface, we estimated a stellar position in the circumstellar envelopes from the distribution of the $\mathrm{SiO}$ masers with a high accuracy. The stellar position was estimated to be $\alpha(\mathrm{J} 2000.0)=07^{\mathrm{h}} 22^{\mathrm{m}} 58^{\mathrm{s}} .3264$, $\delta(\mathrm{J} 2000.0)=-25^{\circ} 46^{\prime} 03^{\prime \prime} .066$.

In addition, we obtained proper motions of the $\mathrm{H}_{2} \mathrm{O}$ masers from multi-epoch VERA observations. We assume that averaged motion of the $\mathrm{H}_{2} \mathrm{O}$ masers represents a stellar traverse velocity.

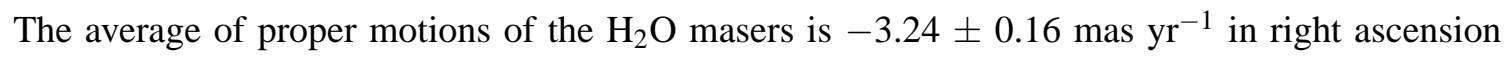

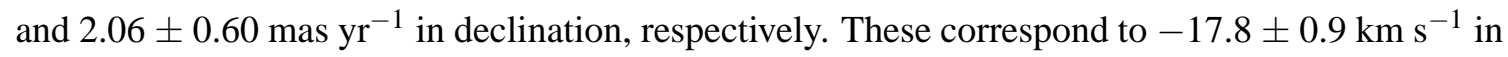
right ascension and $11.3 \pm 3.3 \mathrm{~km} \mathrm{~s}^{-1}$ in declination at the distance of $1.14 \mathrm{kpc}$. The stellar radial
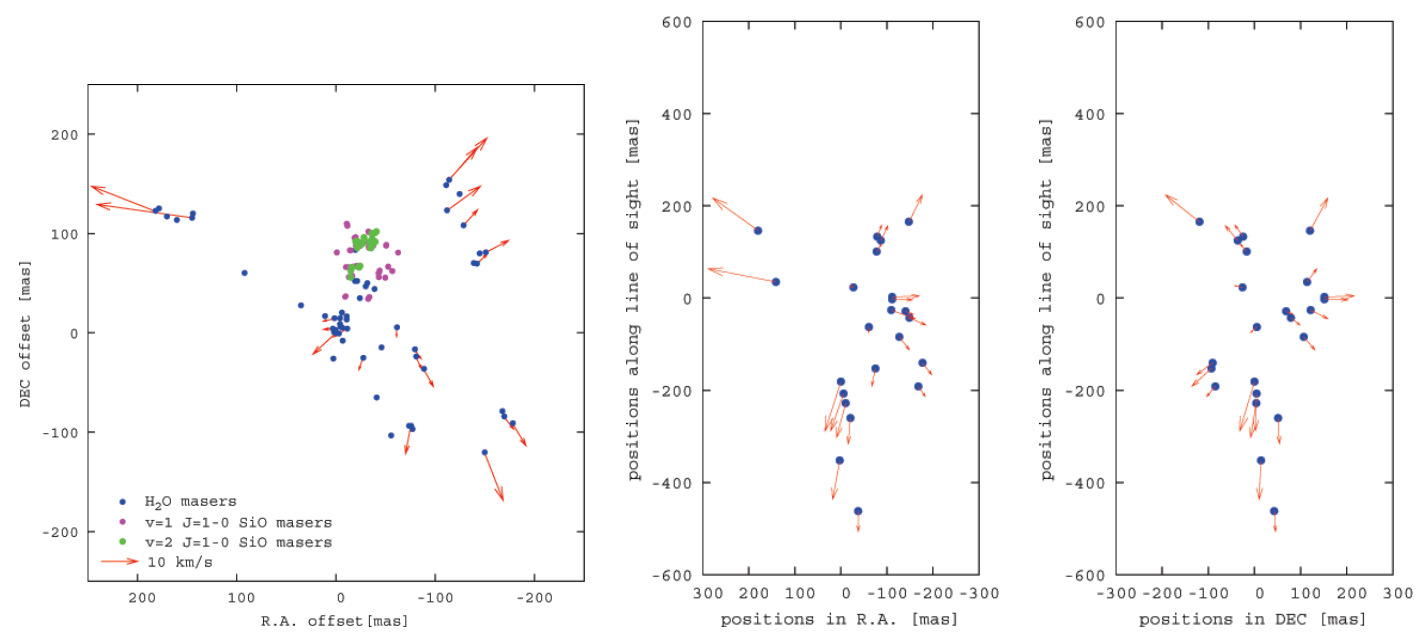

Figure 2: (left) The superposed map of the $\mathrm{H}_{2} \mathrm{O}$ masers (blue points), the $v=1 J=1-0 \mathrm{SiO}$ masers (pink points), and the $v=2 J=1-0 \mathrm{SiO}$ masers (green points). The red arrows represent the absolute motions, which the average motion was subtracted, of the $\mathrm{H}_{2} \mathrm{O}$ maser features. The average motion is $-3.24 \pm 0.16$ mas $\mathrm{yr}^{-1}$ in right ascension and $2.06 \pm 0.60 \mathrm{mas} \mathrm{yr}^{-1}$ in declination, corresponding to $-17.8 \pm 0.9 \mathrm{~km} \mathrm{~s}^{-1}$ and $11.3 \pm 3.3 \mathrm{~km} \mathrm{~s}^{-1}$ at $1.14 \mathrm{kpc}$, respectively. (center) The 2-dimensional positions and velocities of $\mathrm{H}_{2} \mathrm{O}$ maser features projected on $(\mathrm{X}, \mathrm{Z})$ plane. (right) Same as center panel on $(\mathrm{Y}, \mathrm{Z})$ plane. $\mathrm{X}$-, $\mathrm{Y}$ - and Z-axis represent directions of right ascension, declination, and line of sight from the central star, respectively. 
velocity was adopted to be $21.1 \mathrm{~km} \mathrm{~s}^{-1}$ [1]. After the average motion was subtracted, the $\mathrm{H}_{2} \mathrm{O}$ masers show a tendency of expansion from the central star (see figure 2 (left)).

We consider the 3-dimensional structure and kinematics of the circumstellar envelopes around VY CMa. With the 3-dimensional velocities and the 2-dimensional positions on the celestial sphere of the $\mathrm{H}_{2} \mathrm{O}$ maser features, we estimated positions along the line of sight of each maser feature with respect to the central star assuming a simple radial outflow model. Figure 2 show the 3 -dimensional structure and kinematics of the $\mathrm{H}_{2} \mathrm{O}$ masers on projected 2-dimensional planes from our results. It clearly shows elongated structure along the line of sight. According to a previous observational result of $\mathrm{SiO}(v=0 J=1-0)$ emission with VLA [17], VY CMa shows a bipolar outflow along the line of sight. Although we need a more complicated 3-dimensional model including other parameters, for example acceleration, our preliminary results suggest a bipolar outflow of VY CMa along the line of sight, and it is consistent with results from [17]. However, we still cannot explain asymmetric expansion observed with HST in larger scale [10].

\section{Acknowledgment}

This work has benefited from research funding from the European Community's sixth Framework Programme under RadioNet R113CT 20035058187.

\section{References}

[1] Bowers, P. F., Johnston, K. J. \& Spencer, J. H. 1983, ApJ, 274, 733

[2] Chikada, Y., et al. 1991, in Frontiers of VLBI, ed. H. Hirabayashi, M. Inoue, \& H. Kobayashi (Tokyo: Universal Academy Press), 79

[3] Choi, Y. K., et al. 2008, PASJ, 60, 1007

[4] Hachisuka, K. et al. 2006, ApJ, 645, 337

[5] Hirota, T. et al. 2007, PASJ, 59, 897

[6] Hirota, T. et al. 2008, PASJ, 60, 37

[7] Honma, M. et al. 2007, PASJ, 59, 889

[8] Honma, M. et al. 2008, PASJ, 60, 935

[9] Honma, M., Tamura, R., \& Reid, M. J. 2008, PASJ, 60, 951

[10] Humphreys, R. M., Helton, L.A., \& Jones, T. J. 2007, AJ, 133, 2716

[11] Johnson, H. L., Hoag, A. A., Iriarte, B., Mitchell, R. I., and Hallan, K. L. 1961, Lowell Obs. Bull., 5, 133

[12] Kovalev, Y. Y., Petrov, L., Fomalont, E., \& Gordon, D. 2007, AJ, 133, 1236

[13] Lada, C. J., \& Reid, M. J. 1978, ApJ, 219, 95

[14] Le Sidaner, P., \& Le Bertre, T. 1996, A\&A, 314, 896

[15] Massey, P., Leveque, E. M., \& Plez, B. 2006, ApJ, 646, 1203

[16] Meynet, G., \& Maeder, A. 2003, A\&A, 404, 975

[17] Shinnaga, H., Moran, J. M., Young, K. H., and Ho, P. T. P. 2004, ApJ, 616L, 47 
[18] Smith, N., Humphreys, R. M., Davidson, K., Gehrz, R. D. Schuster, M. T., \& Krautter, J. 2001, ApJ, 121, 1111 